\title{
PEV-based P-Q Control in Line Distribution Networks with High Requirement for Reactive Power Compensation
}

\author{
Chenye Wu, Student Member, IEEE, Hossein Akhavan-Hejazi, Student Member, IEEE, \\ Hamed Mohsenian-Rad, Member, IEEE, and Jianwei Huang, Senior Member, IEEE
}

\begin{abstract}
While plug-in electric vehicles (PEVs) are expected to provide economic and environmental benefits to the transportation sector, they may also help the electric grid, both as a potential source of energy storage and as a means to improve power quality and reliability. In this paper, our focus is on the latter, where PEVs offer reactive power compensation using P-Q control at their charger inverters. In this regard, we develop a new optimization-based P-Q control strategy for PEV charging stations to be implemented in line distribution networks that are in great need of reactive power compensation, either because of serving large industrial loads or due to the inductive impact of distribution level wind turbines. Our design is based on a nonlinear power flow analysis, and the design objectives are to perform voltage regulation and demand response. Through various computer simulations, we assess our proposed PEV-based reactive power compensation and compare it with the case where no $P-Q$ control is conducted at PEV charging stations.
\end{abstract}

Keywords-Plug-in electric vehicles, reactive power compensation, P-Q control, power flow analysis, distributed generation.

\section{INTRODUCTION}

Reactive power compensation and voltage control are among the key requirements of power distribution networks to assure power quality and reliability. Traditionally, reactive power compensation is highly needed in areas with large inductive motor loads such as industrial regions. It is also required in presence of wind turbines to support their induction load to avoid generator voltage excursion. Note that, although terminal voltage control is usually supported by the more advanced (and more expensive) industrial-sized wind turbines in large wind farms [1], the smaller wind turbines that are used at the distribution level typically lack such embedded reactive power compensation capabilities. Therefore, voltage control and reactive power compensation continue to remain as two major challenges in power distribution systems, in particular, as distributed generation (DG) units become more popular.

Currently, reactive power compensation is done by a combination of shunt capacitors, switchable capacitors, and static var compensators (SVCs). Unlike shunt capacitors, switchable capacitors and SVCs are capable of adjusting the reactive power compensation level in response to changes in the grid condition, e.g., in case of fluctuations in wind speed. However, they are often considerably more expensive [2]. Therefore, finding alternative options to conduct reactive power compensation for distribution networks is of great practical interests.

C. Wu is with the Department of Electrical and Computer Engineering, Carnegie Mellon University, Pittsburgh, PA, USA, e-mail: wcy@ieee.org. H. Akhavan-Hejazi and H. Mohsenian-Rad are with the Department of Electrical Engineering, University of California, Riverside, CA, USA, emails: \{hossein.akhavan-hejazi, hamed\}@ee.ucr.edu. J. Huang is with the Department of Information Engineering, The Chinese University of Hong Kong, Shatin, New Territories, Hong Kong. e-mail: jwhuang@ie.cuhk.edu.hk. The corresponding author for this paper is H. Mohsenian-Rad.
The possibility of using PEVs to discharge electricity back to the grid has been already widely studied for vehicle-to-grid (V2G) systems, e.g., see [3], [4]. More recently, it has been shown that PEVs may also be capable of offering reactive power compensation, not only in a V2G mode but also during a regular charging cycle, with minimum impact on the battery lifetime [5]-[8]. Therefore, in this paper, our focus is on PEVbased reactive power compensation using P-Q control. In PEVbased P-Q control, the operating mode of the PEV charger inverter is set according to the reactive power compensation and voltage control requirements of the distribution grid, subject to the PEV inverter's operational constraints.

In this paper, our focus is on line distribution networks, c.f. [9], [10]. We consider a scenario where there is a great need for reactive power compensation at the distribution network, due to large industrial loads and distribution level wind turbines that do not have embedded reactive power compensation capabilities. We introduce the system model in Section II. We formulate a PEV-based P-Q control optimization problem based on an accurate nonlinear power flow analysis in Section III, where we also explain how to solve the formulated optimization problem. Through various simulations, in Section IV, we assess our proposed design method and compare it with the case where no P-Q control is conducted at PEV charging stations. The paper is concluded in Section V.

\section{A. Related Works}

There are two types of studies in the literature that are related to this study. First, those papers that use demand response to tackle the intermittency in wind power generation. For example, in [11], Neely et al. used Lyapunov theory to design a centralized queueing system to run delay-tolerant loads using wind power. In [12], He et al. proposed a multiple timescale dispatch to integrate wind generators. In [13], $\mathrm{Wu}$ et al. proposed new pricing schemes to encourage user participation in wind power integration in microgrids. In [14], Li et al. used stochastic programming to coordinate the charging of PEVs based on the amount of wind and solar energy available.

The second group of papers that are related to this study seek to conduct reactive power compensation by means other than shunt capacitors, switchable capacitors, or SVCs. For instance, in [15], Turitsyn et al. considered the possibility of conducting reactive power control at photovoltaic (PV) units. The same idea was further developed in [16]. The use of PEV charger inverters to conduct reactive power compensation was first discussed in [5]. In [17], Cvetkovic et al. showed the possibility of using PEVs for frequency and voltage regulation. A similar idea was also examined in [7] by taking into account the PEVs' charging deadlines. Game theory was used to 


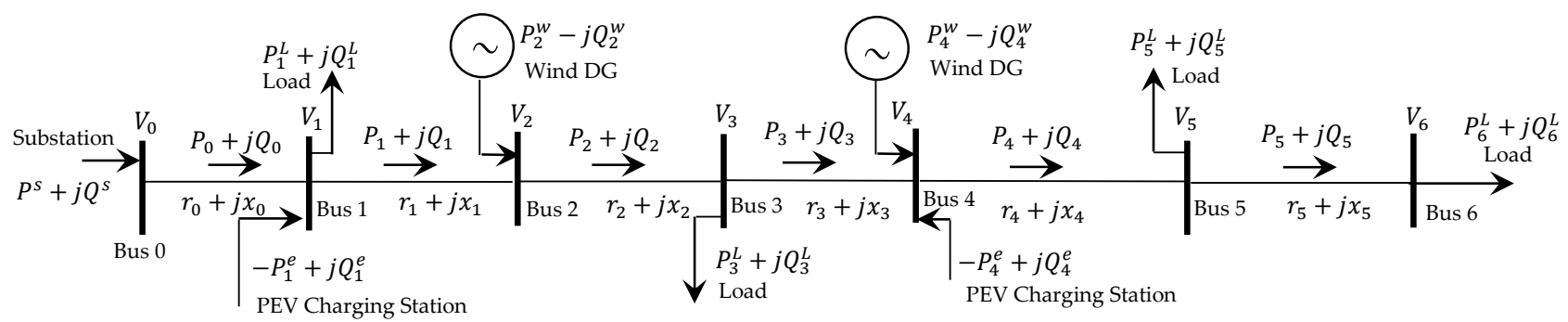

Fig. 1. An example line distribution network with a reference bus, bus 0 , and $B=6$ distribution buses. There are two PEV charging stations at buses 1 and 4 . There are also two wind DG units at buses 2 and 4 . There are background loads at buses 1, 3, 5, and 6 .

encourage PEV participation in reactive power compensation in [8]. A common assumption in [5], [7], [8], [17] is that the exact amount of active or reactive power that is needed from the PEVs are known and given. As a result, the details on the distribution network topology, power flow conditions, the size and location of various types of background loads and DGs are overlooked. We address these shortcomings in this paper.

\section{System Model}

Consider a line distribution network such as the one in Fig. 1 . Let $\mathcal{B}=\{0,1, \cdots, B\}$ denote the set of all buses, where bus 0 is the reference bus that is connected to the substation. Also, let $\mathcal{B}_{P}, \mathcal{B}_{D}$, and $\mathcal{B}_{L}$ denote the sets of buses in which we have PEV charging stations, wind DG units, and background loads, respectively. For the distribution network in Fig. 1, we have $\mathcal{B}=\{0,1, \ldots, 6\}, \mathcal{B}_{P}=\{1,4\}, \mathcal{B}_{D}=\{2,4\}$, and $\mathcal{B}_{L}=$ $\{1,3,5,6\}$. Note that, some buses may belong to multiple sets. For example, $\mathcal{B}_{P} \cap \mathcal{B}_{D}=\{4\}$, because bus 4 is connected to both a PEV charging station and a wind DG unit. For each line between buses $i, i+1 \in \mathcal{B}$, let $P_{i}$ and $Q_{i}$ denote the active and reactive power flow on the line. Also, let $r_{i}$ and $x_{i}$ denote the line resistance and reactance. The voltage phasor at each bus $i \in \mathcal{B}$ is denoted by $V_{i}$ and the current phasor over the line between buses $i, i+1 \in \mathcal{B}$ is denoted by $I_{i}$.

At reference bus 0 , the substation injects complex power $P^{s}+j Q^{s}$ into the distribution feeder, where $P^{s}$ denotes the active power imported from the grid, and $Q^{s}$ indicates the reactive power provided by the shunt capacitor at the substation. At bus $i \in \mathcal{B}_{P}$, the complex power injection from the PEV charging station is denoted by $-P_{i}^{e}+j Q_{i}^{e}$. Note that, PEVs drawn active power to charge their batteries and supply reactive power to contribute in reactive power compensation. At each bus $i \in \mathcal{B}_{D}$, the complex power injection by the wind DG unit is denoted by $P_{i}^{w}-j Q_{i}^{w}$. Note that, wind DG units supply active power but draw reactive power due to their inductive load. The exact values of $P_{i}^{w}$ and $Q_{i}^{w}$ depend on the wind conditions; therefore, they can change from time to time. Finally, at each bus $i \in \mathcal{B}_{L}$, the complex power draw by the background (i.e., non-PEV) load is denoted by $P_{i}^{L}+j Q_{i}^{L}$. Again, the exact values of $P_{i}^{L}$ and $Q_{i}^{L}$ depend on the demand conditions; therefore, they can vary from time to time.

\section{Optimal P-Q Control Design}

The utility company or an aggregator that operates the distribution grid is interested in utilizing the $\mathrm{P}-\mathrm{Q}$ control potential of PEVs towards regulating voltage and performing demand response. In this regard, we assume that the utility constantly monitors the distribution grid conditions and sends appropriate P-Q control commands to the PEV charging stations. For this purpose, the utility needs to solve an optimization problem that takes into consideration the amount of background load at each bus, the amount of power generation at each wind DG bus, the number of plugged-in PEVs at each charging station, charging deadline indicated by each PEV user, and the power flow equations across the distribution network.

\section{A. Power Flow Equations}

In this paper, we use the non-linear power flow equations that were introduced in [9] for radial distribution networks. This model is usually referred to as the DistFlow model [9], [18]. For notational simplicity, at each bus $i \in \mathcal{B}$, we define

$$
v_{i}=\left|V_{i}\right|^{2} \geq 0 \text {. }
$$

Also, for each line between buses $i, i+1 \in \mathcal{B}$, we define

$$
l_{i}=\left|I_{i}\right|^{2}=\frac{\left(P_{i}\right)^{2}+\left(Q_{i}\right)^{2}}{v_{i}} \geq 0 .
$$

Since bus 0 is a reference bus, we have

$$
\begin{aligned}
& v_{0}=1 p . u ., \\
& P_{0}=P^{s}, \\
& Q_{0}=Q_{s} .
\end{aligned}
$$

From (1) and (2) and based on the DistFlow model in [9], at each bus $i \in \mathcal{B} \backslash\{0\}$, we have

$$
\begin{aligned}
& P_{i}=P_{i-1}-r_{i} l_{i}-P_{i}^{e}+P_{i}^{w}-P_{i}^{L}, \\
& Q_{i}=Q_{i-1}-x_{i} l_{i}+Q^{e}-Q_{i}^{w}-Q_{i}^{L}, \\
& v_{i}=v_{i-1}-2\left(r_{i} P_{i-1}+x_{i} Q_{i-1}\right)+\left(r_{i}^{2}+x_{i}^{2}\right) l_{i} .
\end{aligned}
$$

Here, we assume that if $i \notin \mathcal{B}_{P}$, then $P_{i}^{e}=Q_{i}^{e}=0$; if $i \notin \mathcal{B}_{D}$, then $P_{i}^{w}=Q_{i}^{w}=0$; and if $i \notin \mathcal{B}_{L}$, then $P_{i}^{l}=Q_{i}^{l}=0$. Furthermore, we assume that $P_{B}=Q_{B}=0$.

\section{B. PEV Operational Constraints}

Consider a PEV charging station at bus $i \in \mathcal{B}_{P}$. Let $\mathcal{N}_{i}$ denote the set of PEVs in this charging station. For each PEV $n \in \mathcal{N}_{i}$, let $\beta_{n, i}$ denote the departure time, i.e., the time when the PEV must leave the charging station. The departure time is also the charging deadline, i.e., the time by which the PEV's battery must reach its target charging level. At each time $t$, 
the PEV's state-of-charge (SOC) is denoted by $e_{n, i}^{t}$. The target charging level is denoted by $e_{n, i}^{m}$. We assume that the charging time is divided into time slots with an equal length $\Delta T$. The charging rate for each PEV $n$ is updated once every time slot. Let $s$ in kVA denote the PEVs' inverters' maximum apparent power capacity. Now, we are ready to introduce the following constraints for the operation of each PEV $n \in \mathcal{N}_{i}$ :

$$
e_{n, i}^{m}-e_{n, i}^{t} \leq p_{n, i} \Delta T+\left(\beta_{n, i}-t\right) s \Delta T
$$

and

$$
\left(p_{n, i}\right)^{2}+\left(q_{n, i}\right)^{2} \leq s^{2}
$$

where $-p_{n, i}+j q_{n, i}$ denotes PEV $n$ 's complex power injection at bus $i$. Recall from Section II that PEVs draw active power and supply reactive power. At each bus $i \in \mathcal{B}_{P}$, we have

$$
P_{i}^{e}=\sum_{n \in \mathcal{N}_{i}} p_{n, i},
$$

and

$$
Q_{i}^{e}=\sum_{n \in \mathcal{N}_{i}} q_{n, i} .
$$

When using level 1 chargers, $s=1.44 \mathrm{kVA}$; and when using level 2 chargers, $s=7.68 \mathrm{kVA}$. Next, we explain the rational behind (9) and (10). In (9), the left hand side indicates the amount of energy that PEV $n$ needs to draw from bus $i$ to reach its target charging level. On the right hand side, the first term indicates the amount of energy that PEV $n$ draws in the current time slot, given its current charging rate $p_{n, i}$. The second term indicates the maximum amount of energy that PEV $n$ will be able to draw during the upcoming time slots before the charging deadline $\beta_{n, i}$, assuming that it does not provide reactive power. If the inequality in (9) holds, then PEV $n$ is assured to reach its target SOC by or before its charging deadline. In (10), we simply make sure that the operation of PEV n's charger is within its supported capacity range.

\section{Substation Operational Constraints}

Next, we take into account the constraints associated with the substation. Recall from Section II that $P^{s}$ gives the amount of active power that the substation imports from the grid. In practice, this amount is limited by the operational capacity of the substation, which we denote by $\bar{P}$. To avoid overloading the substation transformers, we need to have

$$
0 \leq P^{s} \leq \bar{P}^{s}
$$

\section{Design Objective}

Our design objective is two fold. First, we would like to conduct demand response to lower the cost of electricity that is imported to the distribution network by the substation. We seek to achieve this objective by minimizing a cost function $C\left(P^{s}\right)$, which is a convex and increasing function of the total power imported by the substation into the distribution grid. One popular choice of cost function is [19], [20]:

$$
C\left(P^{s}\right)=a\left(P^{s}\right)^{2}+b P^{s}+c,
$$

where $a>0$ and $b, c \geq 0$ are cost parameters. Second, we would like to conduct voltage regulation to improve power quality. We seek to do this by minimizing a voltage regulation function $f\left(v_{1}, \ldots, v_{B}\right)$. For example, we may have

$$
f\left(v_{1}, \ldots, v_{B}\right)=\max _{i \in \mathcal{B}}\left|v_{i}-v_{0}\right|,
$$

where we seek to minimize the maximum deviation of the voltage magnitude at any grid bus from the reference value.

Based on the above two design objectives, we propose to solve the following optimization problem to coordinate P-Q control across all plugged-in PEVs on the distribution network:

$$
\begin{array}{ll}
\text { minimize } & C\left(P^{s}\right)+\lambda f\left(v_{1}, \ldots, v_{B}\right) \\
\text { subject to } & \text { Eqs. }(1)-(13),
\end{array}
$$

where $\lambda$ is a weighting parameter to control the trade-off between demand response and voltage regulation objectives. The optimization variables are $P_{i}$ and $Q_{i}$ for all $i \in \mathcal{B}, P_{i}^{e}$ and $Q_{i}^{e}$ for all $i \in \mathcal{B}_{P}, p_{n, i}$ and $q_{n, i}$ for all $i \in \mathcal{B}_{P}$ and all $n \in \mathcal{N}_{i}$, $v_{i}$ and $l_{i}$ for all $i \in \mathcal{B}$, and $P^{s}$. Furthermore, the parameters are $P_{i}^{w}$ and $Q_{i}^{w}$ for all $i \in \mathcal{B}_{D}, P_{i}^{l}$ and $Q_{i}^{l}$ for all $i \in \mathcal{B}_{L}$, and $Q^{s}$. These parameters are updated at the beginning of each time slot using the utility's monitoring system. Accordingly, problem (16) needs to be solved based on the new set of collected parameters at the beginning of each time slot.

\section{E. Convex Relaxation}

Problem (16) in its current form is not a convex program. Therefore, it is difficult to solve. The cause of non-convexity in (16) is the strong non-linearity of the equality constraint in (2). Inspired by [16], next, we propose to replace the equality constraint in (2) with an inequality constraint. More specifically, for each $i \in \mathcal{B}$, we propose to replace (2) with

$$
l_{i} \geq \frac{\left(P_{i}\right)^{2}+\left(Q_{i}\right)^{2}}{v_{i}} .
$$

After reordering the terms, the above inequality becomes:

$$
\left(P_{i}\right)^{2}+\left(Q_{i}\right)^{2} \leq l_{i} v_{i}
$$

Unlike the constraint in (2), the above constraint is tractable. In fact, (18) is a rotated quadratic cone constraint that forms a convex set [21]. Therefore, after replacing (2) with (18), the relaxed optimization problem becomes convex:

$$
\begin{array}{ll}
\text { minimize } & C\left(P^{s}\right)+\lambda f\left(v_{1}, \ldots, v_{B}\right) \\
\text { subject to } & (1),(3)-(13),(18),
\end{array}
$$

where the optimization variables and parameters are the same as those in problem (16). In [10], Li et al. proposed several checkable conditions to make sure that the above convex relaxation are perfect. Clearly, if perfect relaxation is achieved, i.e., if the inequality in (17) holds as equality, then solving problem (19) is equivalent to solving the original problem in (16). We will investigate the achievability of perfect relaxation in our simulation studies at the end of Section IV. 
TABLE I

Loads and Wind DG Units Active And Reactive Power Data.

\begin{tabular}{c||c|c||c|c}
\hline \hline Minutes & $P_{L}$ & $Q_{L}$ & $P_{W}$ & $Q_{W}$ \\
\hline 5 & $0.147,0.233$ & $0.025,0.047$ & $0.090,0.109$ & $0.023,0.016$ \\
\hline 10 & $0.147,0.230$ & $0.025,0.046$ & $0.084,0.079$ & $0.022,0.018$ \\
\hline 15 & $0.143,0.225$ & $0.024,0.045$ & $0.102,0.087$ & $0.020,0.019$ \\
\hline 20 & $0.137,0.228$ & $0.023,0.046$ & $0.107,0.093$ & $0.017,0.021$ \\
\hline 25 & $0.138,0.230$ & $0.023,0.046$ & $0.107,0.097$ & $0.019,0.018$ \\
\hline 30 & $0.141,0.235$ & $0.024,0.047$ & $0.099,0.087$ & $0.021,0.019$ \\
\hline 35 & $0.144,0.240$ & $0.024,0.048$ & $0.116,0.107$ & $0.020,0.021$ \\
\hline 40 & $0.147,0.238$ & $0.025,0.048$ & $0.102,0.095$ & $0.023,0.018$ \\
\hline 45 & $0.141,0.235$ & $0.024,0.047$ & $0.096,0.098$ & $0.021,0.022$ \\
\hline 50 & $0.140,0.233$ & $0.023,0.047$ & $0.101,0.095$ & $0.017,0.021$ \\
\hline 55 & $0.137,0.235$ & $0.023,0.047$ & $0.094,0.099$ & $0.021,0.021$ \\
\hline 60 & $0.135,0.238$ & $0.023,0.048$ & $0.107,0.114$ & $0.021,0.020$ \\
\hline \hline
\end{tabular}

\section{Simulation Results}

Consider a line distribution network with $B=10$ buses. For each line between buses $i, i+1 \in \mathcal{B}$, we have $r_{i}=0.02$ and $x_{i}=0.06$. The base apparent power is 1 MVA. We have $\mathcal{B}_{L}=\{6,10\}, \mathcal{B}_{D}=\{5,7\}$, and $\mathcal{B}_{P}=\{4,8\}$. A simulation period of one hour is divided into 12 time slots of length 5 minutes. The loads and wind DG powers at different time slots are selected randomly as shown in Table I. The cost function parameters are $a=0.01, b=0.5$, and $c=0$; and the weighting factor is $\lambda=0.5$. The arrival times, departure times, initial charge levels, and target charge levels for a total of 40 PEVs are generated randomly and shown in Table II. Here, a negative arrival time indicates that the PEV is already plugged in at the beginning of the simulation period; and a departure time greater than 60 indicates that the PEV will remain at the charging station at the end of the simulation period.

First, we examine the bus voltages as shown in Fig. 2. We can see that if no P-Q control is conducted, adding the extra load of PEVs can further lower the already low voltage magnitudes along the distribution line, especially at the end buses. However, once P-Q control is conducted, the voltage profile improves significantly to the extent that at almost all buses we achieve a voltage magnitude that is close to 1 p.u.

Next, we examine the voltage magnitude variations during all 12 time slots. The results are shown in Fig. 3 for bus 7 , which is connected to a wind DG unit. We can see that the voltage fluctuations are minimized when our proposed P$\mathrm{Q}$ control mechanism is used. With P-Q control, the amount of active power drawn and reactive power supplied by three different PEVs that are connected to the first charging station are as in Fig. 4. Note that, the charging periods are coordinated to achieve the best voltage profile that is observed in Fig. 3.

Recall from Section III-E that we used convex relaxation to transform problem (16) into the convex problem in (19). If the perfect relaxation is achieved, i.e., if the inequality in (17) holds as equality, then the solution obtained by solving problem (19) is also optimal to the original problem in (16).
TABLE II

PEVs ARrivals, DePartures, AND Charging Data

\begin{tabular}{cccc||cccc}
\hline \hline \multicolumn{4}{c}{ Charging Station 1 } & \multicolumn{5}{c}{ Charging Station 2} \\
\hline$\alpha_{n}$ & $\beta_{n}$ & $e_{n}^{0}$ & $e_{n}^{m}$ & $\alpha_{n}$ & $\beta_{n}$ & $e_{n}^{0}$ & $e_{n}^{m}$ \\
\hline 0 & 75 & 8.0 & 15.6 & 30 & 65 & 8.6 & 16.2 \\
-10 & 115 & 9.3 & 16.5 & 35 & 80 & 3.6 & 6.7 \\
-10 & 75 & 9.3 & 16.5 & 15 & 40 & 11.8 & 14.5 \\
45 & 115 & 2.3 & 4.2 & 55 & 90 & 1.0 & 1.7 \\
-55 & 70 & 15.0 & 16.5 & -5 & 40 & 8.6 & 13.7 \\
5 & 115 & 7.4 & 14.3 & -50 & 85 & 14.3 & 16.5 \\
25 & 75 & 4.8 & 9.3 & 35 & 75 & 3.6 & 6.7 \\
10 & 40 & 8.0 & 11.8 & 0 & 75 & 8.0 & 15.6 \\
-55 & 35 & 15.0 & 16.5 & -20 & 20 & 10.5 & 13.1 \\
-30 & 10 & 11.8 & 13.1 & 5 & 75 & 7.4 & 14.3 \\
-20 & 25 & 10.5 & 13.7 & 15 & 100 & 6.1 & 11.8 \\
-25 & 30 & 11.2 & 15.0 & -50 & 95 & 14.3 & 16.5 \\
30 & 70 & 4.2 & 8.0 & 0 & 80 & 8.0 & 15.6 \\
40 & 55 & 1.0 & 1.7 & -30 & 65 & 11.8 & 16.5 \\
-50 & 55 & 14.3 & 16.5 & 20 & 110 & 5.5 & 10.5 \\
-20 & 75 & 10.5 & 16.5 & -50 & 75 & 14.3 & 16.5 \\
-30 & 65 & 11.8 & 16.5 & 35 & 80 & 3.6 & 6.7 \\
10 & 70 & 6.7 & 13.1 & -15 & 110 & 9.9 & 16.5 \\
0 & 45 & 8.0 & 13.7 & 0 & 110 & 8.0 & 15.6 \\
10 & 45 & 13.7 & 16.5 & -50 & 20 & 14.3 & 16.5 \\
\hline \hline & & & & & & & \\
\hline
\end{tabular}

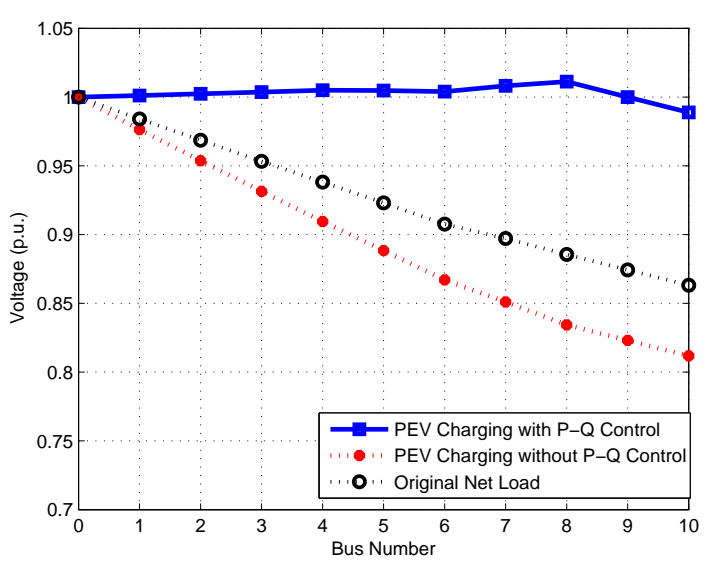

Fig. 2. Voltage profile at all buses during the second simulation time slot.

This issue is investigated in Fig. 5. Here, we can see that the convex relaxation gap is always very low (below $0.03 \%$ ), i.e., we always achieve almost perfect relaxation.

\section{CONClusions}

We proposed a new optimization-based P-Q control strategy for PEV charging stations in line distribution networks. Our design was based on a nonlinear power flow analysis, and our design objectives were to conduct voltage regulation and demand response. Our simulation results showed that the proposed PEV-based reactive power compensation scheme is 


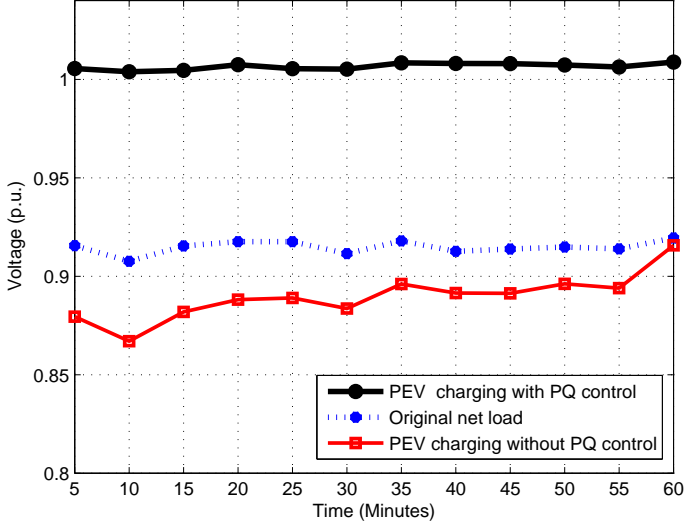

Fig. 3. Voltage variations across different simulation time slots at bus 7 .
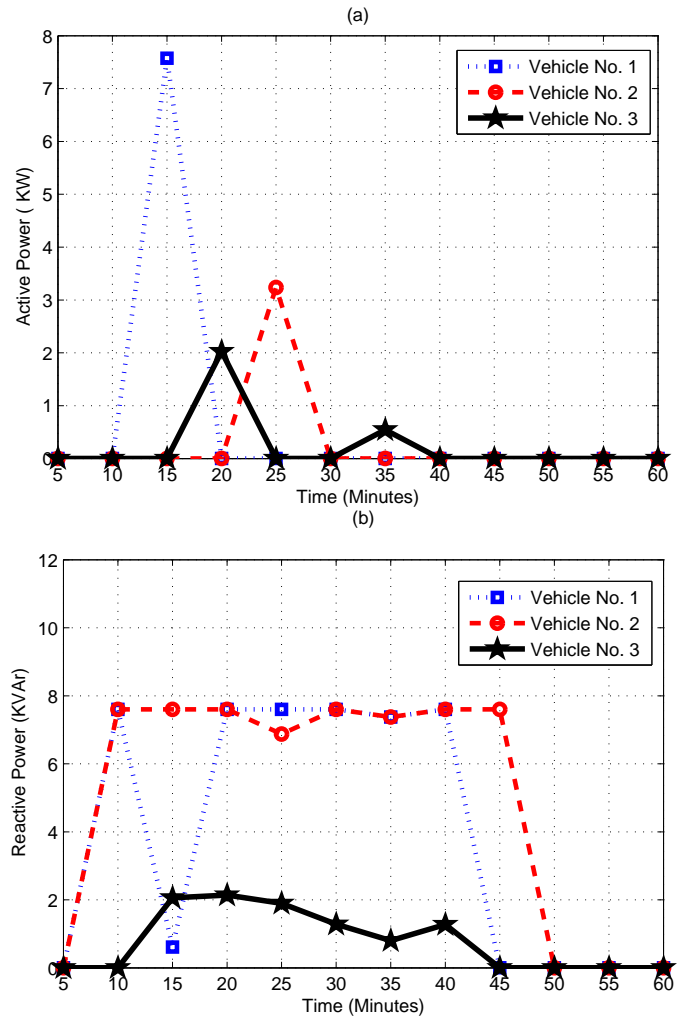

Fig. 4. Operations of three sample PEVs when our proposed design is implemented: (a) Active power draw; (b) Reactive power supply.

very effective in regulating voltage at different buses with different background loads and wind conditions.

\section{REFERENCES}

[1] P. Fairley, "Steady as she blows [wind power, energy storage]," IEEE Spectrum, vol. 40, no. 8, pp. 35-39, 2003.

[2] A. Sode-Yome and N. Mithulananthan, "Comparison of shunt capacitor, SVC and STATCOM in static voltage stability margin enhancement," Int. J. of Electrical Eng. Education, vol. 41, no. 2, pp. 158-171, 2004.

[3] S. Han, S. Han, and K. Sezaki, "Development of an optimal vehicle-togrid aggregator for frequency regulation," IEEE Transactions on Smart Grid, vol. 1, no. 1, pp. $65-72$, june 2010.

[4] C. Wu, H. Mohsenian-Rad, and J. Huang, "Vehicle-to-aggregator interaction game," IEEE Trans. on Smart Grid, vol. 3, no. 1, pp. $434-442$, Mar 2012

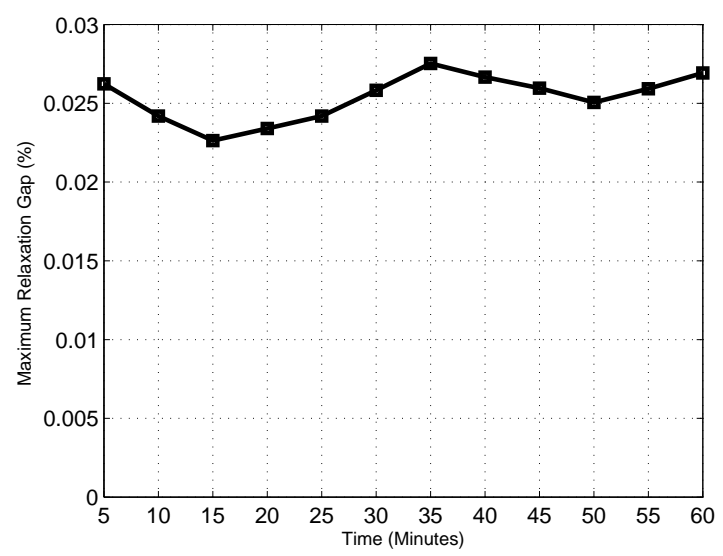

Fig. 5. Maximum convex relaxation gap across all line for different time slots.

[5] M. C. Kisacikoglu, B. Ozpineci, and L. M. Tolbert, "Examination of a phev bidirectional charger system for $\mathrm{v} 2 \mathrm{~g}$ reactive power compensation," in Proc. of the IEEE APEC, feb 2010.

[6] Y. Mitsukuri, R. Hara, H. Kita, E. Kamiya, N. Hiraiwa, and E. Kogure, "Voltage regulation in distribution system utilizing electric vehicles and communication," in Proc. of the IEEE T\&D Conference, may 2012.

[7] C. Wu, H. Mohsenian-Rad, J. Huang, and J. Jatskevich, "PEV-based combined frequency and voltage regulation for smart grid," in Proc. of IEEE ISGT, Washington, DC, Jan. 2012.

[8] C. Wu, H. Mohsenian-Rad, and J. Huang, "PEV-based reactive power compensation for wind dg units: A stackelberg game approach," in in Proc. of IEEE Int. Conf. on Smart Grid Communications, Tainan City, Taiwan, Nov. 2012.

[9] M. Baran and F. Wu, "Optimal sizing of capacitors placed on a radial distribution system," IEEE Trans. on Power Delivery, vol. 4, no. 1, pp. $735-743$, jan 1989.

[10] N. Li, L. Chen, and S. H. Low, "Exact convex relaxation of opf for radial networks using branch flow model," in in Proc. of IEEE Int. Conf. on Smart Grid Communications, Tainan City, Taiwan, Nov. 2012.

[11] M. Neely, A. Tehrani, and A. Dimakis, "Efficient algorithms for renewable energy allocation to delay tolerant consumers," in Proc. of IEEE Int. Conf. on Smart Grid Communications, Gaithersburg, MD, Oct. 2010.

[12] M. He, S. Murugesan, and J. Zhang, "Multiple timescale dispatch and scheduling for stochastic reliability in smart grids with wind generation integration," in Proc. of IEEE INFOCOM, Shanghai, China, April 2011.

[13] C. Wu, H. Mohsenian-Rad, and J. Huang, "Wind power integration via aggregator-consumer coordination: A game theoretic approach," in Prof. of IEEE ISGT, Washington, DC, Jan. 2012.

[14] Y. Li, R. Kaewpuang, P. Wang, D. Niyato, and Z. Han, "An energy efficient solution: Integrating plug-in hybrid electric vehicle in smart grid with renewable energy," in Proc. of IEEE INFOCOM Workshop on Green Networking and Smart Grids, Orlando, FL, Mar. 2012.

[15] K. Turitsyn, P. Sulc, S. Backhaus, and M. Chertkov, "Options for control of reactive power by distributed photovoltaic generators," Proc. of the IEEE, vol. 99, no. 6, pp. 1063 -1073, June 2011.

[16] M. Farivar, C. R. Clarke, S. H. Low, and K. M. Chandy, "Inverter var control for distribution systems with renewables," in Proc. of IEEE Int. Conf. on Smart Grid Communications, Brussels, Belgium, Oct. 2011.

[17] I. Cvetkovic, T. Thacker, D. Dong et al., "Future home uninterruptible renewable energy system with vehicle-to-grid technology," in Proc. of IEEE ECCE, San Jose, CA, Sept. 2009.

[18] M. E. Baran and F. F. Wu, "Optimal capacitor placement on radial distribution systems," IEEE Trans. on Power Delivery, vol. 4, no. 1, pp. $725-734,1989$.

[19] H. Mohsenian-Rad, V. Wong, J. Jatskevich, R. Schober, and A. LeonGarcia, "Autonomous Demand Side Management Based on GameTheoretic Energy Consumption Scheduling for the Future Smart Grid," IEEE Trans. on Smart Grid, vol. 1, no. 3, pp. 320-331, Dec. 2010.

[20] N. Li, L. Chen, and S. H. Low, "Optimal demand response based on utility maximization in power networks," in Proc. of IEEE PES General Meeting, Detroit, MI, Jul. 2011.

[21] R. A. Jabr, "Radial distribution load flow using conic programming," IEEE Trans. on Power Systems, vol. 21, no. 3, pp. 1458-1459, 2006. 